\title{
Forschungsbereiche der Datenökonomie
}

\author{
Durch die rasant steigende Datenmenge ergeben sich viele Möglichkeiten, neue \\ datengetriebene Geschäftsmodelle zu entwickeln. Die Datenökonomie gewinnt immer \\ mehr an Bedeutung. Damit das ökonomische Potenzial ausgeschöpft werden kann, ist es \\ elementar, dass genügend Fachkräfte verfügbar sind. Wichtige Forschungsbereiche der \\ Datenökonomie sind die Bedeutung von Kooperationen und Wissen für die Entwicklung neuer \\ Geschäftsmodelle, Daten und Algorithmen sowie das Branchencluster des Gesundheitswesens.
}

\begin{abstract}
Die Digitalisierung im wirtschaftlichen, öffentlichen und privaten Bereich ist unmittelbar mit einer immer größeren Datenmenge verbunden. Als Paradebeispiel für datengenerierende Prozesse werden für den Wirtschaftsbereich oft die vernetzten Maschinen einer Industrie-4.0-Produktionsstätte genannt. Im privaten Bereich sind es die Interaktionen über soziale Netzwerke oder die Nutzung von Wearables (mit Sensorik ausgestattete Kleidung). Diese Beispiele bilden jedoch nur einen Bruchteil der digitalisierten Prozesse der heutigen Zeit ab. Die Digitalisierung führt zu einem massiven Zuwachs von Daten in allen Bereichen: So wurden 2018 weltweit Daten in einem Umfang von 33 Zettabyte (Trillionen Gigabyte) generiert. ${ }^{1}$ Erwartet wird, dass sich dieser Wert bis 2025 auf etwa 175 Zettabyte mehr als verfünffacht. ${ }^{2}$
\end{abstract}

(C) Der/die Autor(en) 2020. Open Access: Dieser Artikel wird unter der Creative Commons Namensnennung 4.0 International Lizenz (http:// creativecommons.org/licenses/by/4.0/deed.de) veröffentlicht.

Open Access wird durch die ZBW - Leibniz-Informationszentrum Wirtschaft gefördert

1 Vgl. D. Reinsel, J. Gantz, J. Rydning: The Digitization of the World. From Edge to Core, IDC White Paper, Framingham, USA, 2018.

2 Vgl. ebenda.

Can Azkan ist wissenschaftlicher Mitarbeiter in der Abteilung Datenwirtschaft am Fraunhofer-Institut für Software- und Systemtechnik (ISST) in Dortmund.

Dr. Henry Goecke leitet die Forschungsgruppe Big Data Analytics im Institut der deutschen Wirtschaft in Köln.

Markus Spiekermann leitet die Abteilung Datenwirtschaft am ISST.
Mit einer immer größeren Datenmenge und fortschreitenden technischen Möglichkeiten zur Sammlung, Speicherung und Analyse dieser Daten steigt auch die volkwirtschaftliche Bedeutung von allen Geschäftsmodellen, die auf Daten basieren. Neben den Daten selbst wird die Fähigkeit, technologische Möglichkeiten in notwendigem Maße einsetzen zu können, zu einem zentralen Wettbewerbsfaktor für Unternehmen und die Gesamtwirtschaft. $\mathrm{Zu}$ solchen Möglichkeiten zählt, neben vielen anderen, beispielsweise künstliche Intelligenz, der 3D-Druck oder auch die Distributed-Ledger-Technik. Ob Unternehmen die Fähigkeit zum Einsatz solcher technologischen Möglichkeiten besitzen, wird durch zahlreiche Faktoren determiniert. Diese reichen von Finanzierungsmöglichkeiten über die Verfügbarkeit von Fachkräften (hier ist sowohl die Zahl der Personen als auch deren passende Qualifikation bedeutsam) bis hin zu gesetzlichen Regularien.

Die technischen Möglichkeiten in Kombination mit der immer größer werdenden Datenmenge bergen großes ökonomisches Potenzial. Die Anwendungsmöglichkeiten erlauben die Entwicklung ganz neuer, datengetriebener Geschäftsmodelle. Allgemein sind die Nutzung, Auswertung und Verknüpfung von Daten als Innovationsbeschleuniger einzustufen. Die Auswirkungen der fortschreitenden Digitalisierung induzieren durch die Omnipräsenz von Digitalisierung, Daten und künstlicher Intelligenz zudem zahlreiche gesellschaftliche und politische Ableitungen. Der Begriff der Datenökonomie fasst, grob gesprochen, die gesamte wirtschaftliche Aktivität unter Verwendung von Daten zusammen.

\section{Datengetriebene Geschäftsmodelle}

Die Digitalisierung hat dazu geführt, dass sich vermehrt digitale und datengetriebene Geschäftsmodelle entwickeln. Eine einheitliche Abgrenzung zu klassischen Geschäftsmodellarten existiert nicht. Datengetriebene Geschäftsmodelle sind eine Unterkategorie der digitalen Geschäftsmodelle. Ein datengetriebenes Geschäftsmodell zeichnet sich 
insbesondere dadurch aus, dass Daten eine Schlüsselfunktion bei der Wertschöpfung einnehmen. ${ }^{3}$ Dies bedeutet nicht, dass in einem datengetriebenen Geschäftsmodell die Wertschöpfung ausschließlich auf Daten basiert. Die Neukonzeption eines Produktes oder einer Dienstleistung ist eine Variante eines datengetriebenen Geschäftsmodells. Daneben kann sich ein datengetriebenes Geschäftsmodell auch in der Optimierung oder der Erweiterung eines bestehenden Geschäftsmodells darstellen.

Die Bedeutung von datengetriebenen Geschäftsmodellen für die Wettbewerbsfähigkeit und die Produktivität von Unternehmen ist vielfach nachgewiesen. Beispielsweise lässt sich belegen, dass die datengestützte Entscheidungsfindung in Unternehmen deren Produktivität um gut $5 \%$ steigert. ${ }^{4}$ Im Kontext der datengetriebenen Geschäftsmodelle wird vermehrt die Bezeichnung Datenökonomie verwendet. Dieser Begriff wurde für eine Studie für die Europäische Kommission wie folgt definiert: ${ }^{5}$ „Die Datenökonomie misst die Gesamtauswirkungen des Datenmarktes auf die Gesamtwirtschaft. Sie umfasst die Erzeugung, Sammlung, Speicherung, Verarbeitung, Verteilung, Analyse, Aufbereitung, Bereitstellung und Nutzung von Daten, die durch digitale Technologien ermöglicht werden. Die Datenwirtschaft umfasst auch die direkten, indirekten und induzierten Auswirkungen des Datenmarktes auf die Wirtschaft" 6 . Datenökonomie ist somit weiter gefasst als das Konzept der datengetriebenen Geschäftsmodelle, die aber einen wesentlichen Bestandteil der Datenökonomie darstellen. Datengetriebene Geschäftsmodelle stehen für eine eher betriebswirtschaftliche Perspektive, während die Datenökonomie einen eher volkswirtschaftlichen Blickwinkel hat.

\section{Ökonomische und politische Bedeutung der Datenökonomie}

Zahlreiche Studien belegen die volkswirtschaftliche Bedeutung der Datenökonomie und insbesondere die Erwartung, dass sich diese rasant ausweiten wird. ${ }^{7}$ Von

3 Vgl. P. Hartmann et al.: Big Data for Big Business? A Taxonomy of Data-driven Business Models used by Start-up Firms, Cambridge, Großbritannien, 2014.

4 Vgl. E. Brynjolfsson, L. M. Hitt, H. H. Kim: Strength in Numbers: How Does Data-Driven Decisionmaking Affect Firm Performance?, in: SSRN Electronic Journal, April 2011.

5 Vgl. auch Europäische Kommission: Building a European data economy, https://ec.europa.eu/digital-single-market/en/policies/building-european-data-economy (8.10.2019). Für weitere Definitionen vgl. Digital Realty: Data Economy Report, San Francisco 2018; vgl. Bundesverband Digitale Wirtschaft (BVDW): Data Economy. Datenwertschöpfung und Qualität von Daten, Berlin 2018; vgl. D. Möller, A. Karstedt-Meierrieks: Rechtliche Aspekte der Datenökonomie, Thesenpapier, Berlin 2017.

6 Vgl. International Data Corporation (IDC): Open Evidence: European Data Market SMART 2013/0063. Final Report (8.10.2019).

7 Vgl. Bundesverband Digitale Wirtschaft e.V.: Data Economy. Datenwertschöpfung und Qualität von Daten, Berlin 2018.
Abbildung 1

Wachstum der Beschäftigung in der Datenökonomie

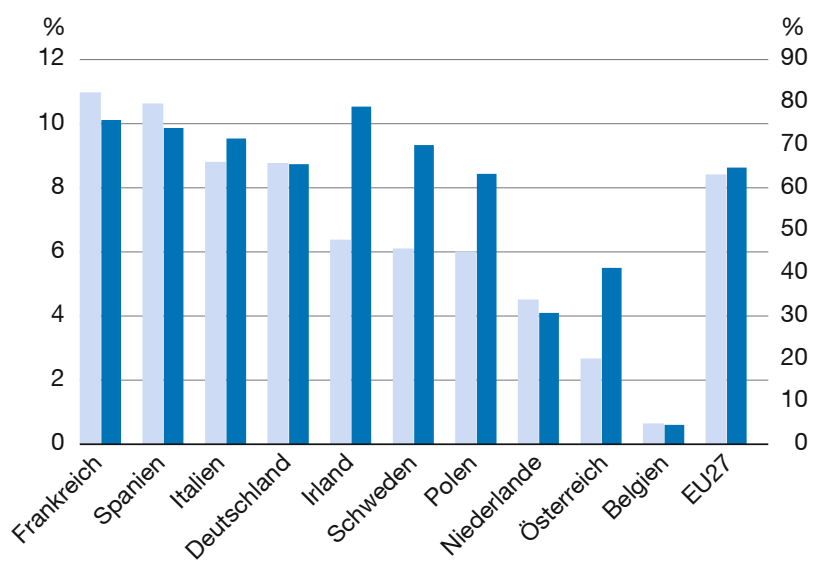

- Veränderung von 2017 bis 2018 (linke Skala)

- Veränderung von 2018 bis 2025 (rechte Skala)

Top-10-Länder in der EU27 nach der Größe des nominalen Bruttoinlandsprodukts 2018

Quelle: International Data Corporation (IDC) 2019.

2017 bis 2018 stieg das Volumen der Datenökonomie in den EU27-Staaten (EU28 ohne Großbritannien) um gut $12 \%$ von knapp 267 Mrd. auf gut 300 Mrd. Euro. ${ }^{8}$ In drei unterschiedlichen Szenarien (Herausforderungs-, Basis- und dynamisches Wachstumsszenario) wird berechnet, dass die Bedeutung der Datenökonomie im Vergleich zum aktuellen Wert von 2018 bis 2025 um den Faktor 1,5, 1,8 oder sogar 2,7 zunehmen wird. Die Szenarien unterscheiden sich hinsichtlich der angenommenen Geschwindigkeit, mit der sich datenbasierte Innovationen durchsetzen, der Größe ihrer Auswirkung auf das gesamtwirtschaftliche Wachstum sowie der Konzentration der Markmacht in Bezug auf den Zugang, die Kontrolle und die Auswertung von Daten.

Ähnlich dynamisch stellt sich die Entwicklung am Arbeitsmarkt in der Datenökonomie dar (vgl. Abbildung 1). In der gesamten EU wuchs die Zahl der Beschäftigten in der Datenökonomie von 2017 bis 2018 um mehr als $8 \%$. Besonders dynamisch war diese Entwicklung in Irland und in Frankreich mit Wachstumsraten von über $10 \%$. Deutschland liegt mit 8,7\% nahezu gleichauf mit dem Durchschnittswert der EU27 von 8,6\%. Auch zukünftig wird erwartet, dass der Arbeitsmarkt in der Datenökonomie weiter dynamisch wächst. In einem Basisszenario wird bis 2025 von einem weiteren

8 Vgl. IDC Italia, The Lisbon Council: Second Report on Facts and Figures. Updating the European Data Market Monitoring Tool, Update of the European Data Market Study SMART 2016/0063 (8.10.2019). 
Beschäftigungswachstum von im Schnitt über $60 \%$ in der EU27 ausgegangen. Spitzenreiter sind Frankreich und Spanien mit erwarteten Wachstumsraten von etwa $80 \%$. Allerdings wird davon ausgegangen, dass diese Fachkräftenachfrage auf dem Arbeitsmarkt nicht von dem zukünftigen Angebot gedeckt werden kann. Die Fachkräftelücke lässt sich für die EU27 im Jahr 2018 bereits auf knapp eine halbe Million Stellen beziffern. Im Basisszenario wird angenommen, dass sich diese Lücke für die EU27 bis 2025 nahezu verdoppelt und auf über 900000 Personen steigt. ${ }^{9}$

Neben den ökonomischen Aspekten spielt die Datenökonomie auch politisch eine große Rolle. Dies spiegelt sich insbesondere darin wider, dass in zahlreichen Ländern an der strategischen Ausrichtung von Politik, Wirtschaft und Gesellschaft gearbeitet wird. Dies bezieht sich beispielsweise auf Digitalisierungsstrategien, Strategien zur Nutzung von künstlicher Intelligenz oder auch die Initiative der Europäischen Kommission zum Aufbau einer Europäischen Datenwirtschaft. So haben Deutschland und viele andere Länder, aber auch die Europäische Union, insgesamt in den letzten Jahren z. B. konkrete Strategien für die Nutzung von künstlicher Intelligenz vorgelegt. ${ }^{10}$ Die Datenökonomie ist ein bestimmendes Thema, das auch die politischen Aktivitäten in Deutschland und Europa umfassend beeinflusst.

\section{Forschung im Bereich der Datenökonomie}

Auch in der Forschung gewinnt das Thema der Datenökonomie an Bedeutung. Hierbei ist insbesondere die Innovationstätigkeit im Bereich der Datenökonomie volkswirtschaftlich relevant. Zwischen Forschungsinstitutionen und Unternehmen gibt es Spill-Over-Effekte, die bewirken, dass Wissen von der Forschung in die Wirtschaft transferiert wird. Dies manifestiert sich unter anderem in Innovationen, die in Form von neuen Produkten und Dienstleistungen zu einer erhöhten ökonomischen Aktivität führen. Zur Analyse der Innovationsbereiche im Kontext der Datenökonomie wurde die Datenbank Scopus verwendet, auf der über 70 Mio. wissenschaftliche Veröffentlichungen zu finden sind, die im Peer-Review-Verfahren qualitätsgesichert wurden. ${ }^{11}$ Zum Auffinden der relevanten Veröffentlichungen wurde nach Stichwörtern wie "digital economy“, „data economy“, „digital business model“, „data driven business model“, „digital business“, „digital platforms“, „data

9 Ebenda.

10 Vgl. Bundesregierung: Strategie Künstliche Intelligenz der Bundesregierung, Stand: November 2018.

11 Vgl. Elsevier: Scopus, https://www.elsevier.com/solutions/scopus (14.10.2019). technologies“, „digital disruption“, „,digital transformation" in Verbindung mit „innovation" gesucht. ${ }^{12}$ Als Ergebnis wurden 860 relevante Veröffentlichungen im Bereich der Datenökonomie identifiziert.

Es zeigt sich, dass die Zahl innovationsgetriebener wissenschaftlicher Publikationen im Bereich der Datenökonomie absolut betrachtet zunimmt. Um dies zu adressieren, wurde die wissenschaftliche Literatur gezielt nach Problemen, Barrieren und Herausforderungen durchsucht, um erste Hinweise darauf zu erhalten, in welchen Bereichen in Zukunft Innovationen zu erwarten sind. Deutschland liegt im internationalen Vergleich bei der Zahl der Aufsätze direkt hinter den USA auf Platz 2, noch vor Großbritannien. Wird der Inhalt dieser $860 \mathrm{Pu}-$ blikationen mithilfe von Text Mining genauer analysiert, zeigen sich im Wesentlichen neun Innovationscluster im Bereich der Datenökonomie: Cooperation, Economics, Knowledge-Based-Systems, Big Data, Decision-Making, Digital Transfomation, Collaborative Network, Information Management und Healthcare (vgl. Abbildung 2). Die Cluster stehen jeweils für einen thematischen Schwerpunkt der Forschung zur Datenökonomie.

Vier Schwerpunkte sind dabei besonders hervorzuheben:

1. Kooperationen (Cluster Cooperation und Collaborative Network);

2. Wissen (Cluster Knowledge-Based-Systems und Information Management) für die Entwicklung von neuen Geschäftsmodellen;

3. Daten und Algorithmen (Cluster Big Data und Decision Making) beinhaltet die Generierung, Verarbeitung, Speicherung und Handhabung bis hin zur Auswertung von Daten durch die Anwendung von Algorithmen wie Machine Learning oder der Mustererkennung;

4. das Branchencluster im Gesundheitswesen ist das einzige Cluster mit einem Branchenfokus. Dies verdeutlicht zum einen, dass die Nutzung von Daten und Informationen im Gesundheitswesen äußerst relevant ist. Zum anderen lässt sich ableiten, dass Forschung und Entwicklung in diesem Bereich vergleichsweise hoch sind.

12 Vgl. C. Azkan, M. Spiekermann, H. Goecke: Uncovering research streams in Data Economy using text mining algorithms, Florenz 2019. Die folgenden Ergebnisse beziehen sich auf diese Publikation. 
Abbildung 2

Clusteranalyse: Forschungsschwerpunkte in der Datenökonomie

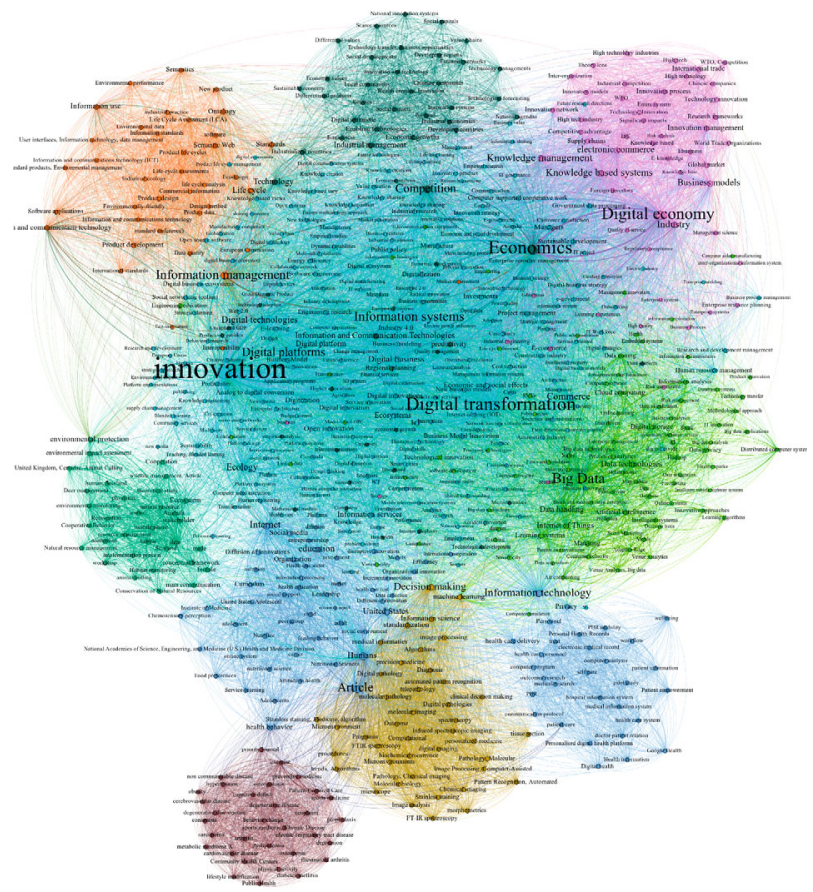

Quelle: C. Azkan, M. Spiekermann, H. Goecke: Uncovering research streams in Data Economy using text mining algorithms, ISPIM Conference, Florenz 2019.

\section{Herausforderungen für die Datenökonomie}

Im Zuge der Digitalisierung wird die Datenökonomie immer wichtiger. Um die Wettbewerbsfähigkeit deutscher und europäischer Unternehmen in Bezug auf die Datenökonomie zu erhalten und zu verbessern, ist es notwendig, in ausreichendem Umfang Fachkräfte zur Verfügung zu stellen und die relevanten Innovationsbereiche zu identifizieren. Insgesamt zeigen sich vier Schwerpunkte, die im Bereich der Datenökonomie wissenschaftlich bearbeitet werden: Kooperationen und
Wissen für die Entwicklung von neuen Geschäftsmodellen, Daten und Algorithmen sowie das Branchencluster des Gesundheitswesens. Als Innovationsbereiche können darüber hinaus Umgang und Handhabung von Daten, Entwicklung von Algorithmen zum maschinellen Lernen sowie Entscheidungsfindung auf Basis von Daten identifiziert werden. ${ }^{13}$

Die Aufgabe für Forschung und Unternehmen besteht zudem darin, Hürden und Hemmnisse für jeden einzelnen Innovationsbereich herauszuarbeiten, denen sich Wirtschaft und Forschung gegenübersehen. Basierend auf den Ergebnissen sollten dann individuelle Handlungsempfehlungen abgeleitet werden, wie die jeweiligen Innovationsbereiche gestärkt werden können. In einigen Bereichen gibt es dazu bereits erste Ansätze: So wurden beispielsweise im Bereich Big Data insbesondere fehlendes Know-how, unzureichende Datenqualität sowie ein fehlendes Budget in Unternehmen als Hemmnisse für den Einsatz von Big Data identifiziert. ${ }^{14}$

Neben der spezifischen Analyse von Innovationsbereichen und den sich daraus ableitenden Potenzialen ist es übergeordnet eine notwendige, wenn auch nicht hinreichende Bedingung für die Teilhabe an der Datenökonomie, dass Unternehmen befähigt werden, ihre Daten als Ressource zu erkennen und effektiv sowie effizient bewirtschaften zu können. In diesem Zusammenhang sind in der Grundlagenforschung zahlreiche Themen zu bearbeiten, zu denen unter anderem Data Governance und die Bewertung von Daten als Wirtschaftsgut gehören. Auch zahlreiche juristische Fragen bleiben zu klären. Wichtig ist grundsätzlich, dass neben der Analyse und Ergebnisaufbereitung aus dem wissenschaftlichen Kontext heraus auch stets der Transfer in die Praxis erfolgt, der für die Entwicklung der Datenökonomie von immenser Bedeutung ist.

13 Ebenda.

14 Vgl. B. Engels, H. Goecke: Big Data in Wirtschaft und Wissenschaft. Eine Bestandsaufnahme, Köln 2019.

Title: Research Areas of Data Economics

Abstract: In the course of advancing digitisation in almost all areas of life, the amount of data is increasing rapidly, resulting in numerous possibilities for developing new data-driven business models. The data economy is becoming more and more important. In order to exploit the economic potential of the data economy, it is essential to have enough data professionals available. Current research topics in data economy include the importance of cooperation and knowledge for the development of new business models, the area of data and algorithms as well as the health care sector.

JEL Classification: C38, 030, 032 